Check for updates

Cite this: RSC Adv., 2017, 7, 37402

Received 4th March 2017 Accepted 20th June 2017

DOI: $10.1039 / c 7 r a 02668 d$

rsc.li/rsc-advances

\section{Local structural studies on Co doped ZnS nanowires by synchrotron X-ray atomic pair distribution function and micro-Raman shift $\dagger$}

\author{
U. P. Gawai and B. N. Dole (DD *
}

Micro-flowers consisting nanowires of pure and cobalt-doped ZnS samples were synthesized via a hydrothermal method. The atomic structures of the obtained nanowires (NWs) were studied by X-ray atomic pair distribution function (PDF) analysis and total synchrotron $X$-ray scattering. The PDF method was used to describe the wurtzite structure of the as-synthesized samples and the results suggested that a cation-cation $\left(\mathrm{Zn}^{2+}-\mathrm{Zn}^{2+}\right)$ distance of 3.8343-3.7733 $\AA$ with a coordination number $\sim 4$ for the wurtzite structure. The diameters of the samples were evaluated using the PDF data and were in good agreement with the TEM results. Raman spectra of the Co-doped ZnS NWs exhibited a first-order phonon mode at 349.34, 347.08, and $344.70 \mathrm{~cm}^{-1}$, corresponding to the $A_{1} / E_{1}$ longitudinal-opticalphonon vibration mode, in addition to the strong surface optical (SO) phonon mode observed at 330 and $333 \mathrm{~cm}^{-1}$. In the Raman spectra, the peak position of the longitudinal-optical-phonon plasmon coupled mode was shifted to higher frequencies with increasing the cobalt concentration. It was observed that the energy band gap kept on decreasing with increases in the cobalt content. XPS analysis revealed that the chemical state and $\mathrm{S}$ vacancies could be controlled by varying the amount of cobalt content in the NWs. The FTIR spectra demonstrated that the sharp peaks were mainly due to all manner of bending vibrations within the NWs observed in the fingerprint region $\left(400-1500 \mathrm{~cm}^{-1}\right)$. The peaks observed at 1038.5, 1163; 1027, 1150;1011, 1150;1019.4, $1133 \mathrm{~cm}^{-1}$; and $1011,1158 \mathrm{~cm}^{-1}$ were due to the formation of a microstructure for the pure and Co-doped ZnS samples, respectively.

\section{Introduction}

The diluted magnetic semiconductor (DMS) materials II-VI, such as CdS, CdSe, ZnS, and ZnSe, have been the subject of intensive research because their energy band gap falls into the visible spectrum and can be tunable with transition metals when the particle size is reduced below $\sim 10 \mathrm{~nm}^{\mathbf{1 , 2}}$ In recent years, semiconductor nanomaterials have attracted significant interest due to their unique properties, which are different from those of the bulk materials. Nowadays, sulfide DMSs have attracted significant attention due to their versatile and novel potential application in electronics and optoelectronic, because of their direct band gap. $\mathrm{ZnS}$ is one of the most well-known host materials for efficient phosphors. ZnS is an impotent DMS material with a direct wide band gap of $3.77 \mathrm{eV}$ for its wurtzite

Advanced Materials Research Laboratory, Department of Physics, Dr. Babasaheb Ambedkar Marathwada University, Aurangabad-431 004, India. E-mail: drbndole. phy@gmail.com

$\dagger$ Electronic supplementary information (ESI) available: XPS and EDAX spectra were provided in Fig. S1 and S2 for details information. Also the full scan SCXRPD was provided in Fig. S3 with tabulated values in Tables S1 and S2 for pure and Co doped ZnS NWs. The first peak fitted with Gaussian, first PDF peaks width and strain in $\mathrm{Zn}-\mathrm{S}$ bond given in Fig. S4. See DOI: 10.1039/c7ra02668d structure, ${ }^{3}$ and $3.72 \mathrm{eV}$ for its cubic ${ }^{4}$ structure at $300 \mathrm{~K}$ together with a large exciton binding energy $(\sim 40 \mathrm{meV})$. ZnS has been used as a base material for cathode ray tubes (CRTs) and field emission display (FED) phosphors, ${ }^{5,6}$ electroluminescent devices, light emitting diodes (LEDs), ${ }^{7}$ infrared (IR) window, ${ }^{\mathbf{8}}$ field effect transistors (FETs), ${ }^{9}$ and gas and photosensors. ${ }^{\mathbf{1 0 , 1 1}}$

In the last two decades, much attention has been focused on the synthesis, physical properties, and applications of onedimensional (1D) nanostructured materials, such as nanorods, ${ }^{12,13}$ nanowires, ${ }^{14-16}$ nanoribbons, ${ }^{17}$ and nanotubes. ${ }^{18}$ For the preparation of $1 \mathrm{D}$ materials, there are various effective techniques, such as the hydrothermal method, ${ }^{16,19-22}$ thermal evaporation method, ${ }^{23}$ pulse laser deposition, ${ }^{24}$ electrochemical deposition, ${ }^{25}$ molecular beam epitaxy (MBE), ${ }^{26}$ microwave assisted technique, ${ }^{27}$ and chemical vapor deposition. ${ }^{28}$

$\mathrm{ZnS}$ is also applicable in photocatalysis because of its electron-hole pair generation capability by photoexcitation without any co-agent. The cubic (sphalerite) ZnS structure can be obtained under mild conditions and is very stable at room temperature. The hexagonal $\mathrm{ZnS}$ structure has been obtained at very high temperature, but very few reports show it at low temperature. ${ }^{29,30}$ The determination of the crystal structure (CS) is a key part of materials science. For the purpose of determining the structure and microstructure of crystalline solids, 
the powder diffraction method is widely used. It is an excellent and existing challenge for the determination of local structure of complex materials. Nowadays, synchrotron X-ray powder diffraction (SCXRPD) with fast computing methods has been used for the determination of the atomic-scale structure of materials. SCXRPD with the pair distribution function (PDF) is one of the most powerful tools to attain the local structure of atoms with shorter length scales. ${ }^{31,32}$ PDF gives a histogram of interatomic separations in a nanomaterial and represents a weighted bond length distribution. SCXRPD is used for the determination of the crystal structure of nanocrystals, whereby it acts as a grating system and produces a diffraction pattern (Braggs peaks) of the crystal structure. Bragg peaks arise due to the translational symmetry present in the samples. Recently, rapid developments in nanoscience and nanotechnology have posed new challenges for the study of atomic-scale structures using SCXRPD. Owing to the nanocrystalline nature of certain materials, it does not always act as a perfect grating, therefore the SCXRPD patterns can show both Bragg peaks and diffuse components. ${ }^{31}$ PDF uses both the Bragg peaks and diffuse components to reveals the total scattering in the SCXRPD data. PDF is obtained from a total scattering powder diffraction pattern via Fourier transform analysis. Since the total scattering pattern is composed of Braggs as well as diffuse scattering contributions, PDF contains local, medium-range, and longrange structural information. PDF reveals three types of information about a pair of atoms in the structure: (i) the peak position contributes to the average distance separation of the pair; (ii) the integrated intensity of each peak reveals the number of coordinate atoms; and (iii) the width and shape of the peak indicate the static or dynamic disorder in the pair. ${ }^{32}$

Raman spectroscopy is a vibrational technique routinely used to provide local structural information at the atomic scale. Nanostructure materials with a large surface to volume ratio and local surface modulation lead to the observation of an extraordinary phonon mode known as the surface optical (SO) phonon mode, which is not observed for their bulk counterparts. ${ }^{33}$ Recently, longitudinal-optical-phonon and plasmon coupling (LOPC) were investigated using Raman scattering to characterize the hole as well as electron concentrations in GaMnAs and ClZnSe, respectively. ${ }^{34,35}$ The strong interaction between LO phonons and collective excitation plasmons via their associated macroscopic electric fields has also been extensively studied in other compound semiconductors, such as SiC, GaAs, and GaN. ${ }^{36}$

In this paper, we applied the PDF method to study the local structure, size, and strain in pure and Co-doped $\mathrm{ZnS}$ nanowires as a function of the nanowire diameters. The core structure of the studied nanowire can be described by a mixed crystalline structure intermediate between zinc blende and wurtzite. In the Raman spectra, the LOPC mode for the samples was attributed to the existence of free electrons by $\mathrm{Co}^{+}$ions. The energy band gap kept on decreasing with an increasing cobalt content. XPS analysis revealed the chemical state and $\mathrm{S}$ vacancies, which could be controlled by varying the amount of cobalt content in the NWs. Hence, we undertook to tackle this problem to investigate the diverse facets of Co-doped ZnS NWs. We report herein on the different studies we carried out, such as SCXRPD,
PDF, TEM, FE-SEM, micro-Raman, XPS, FTIR, and UV-Vis into the Co-doped ZnS NWs.

\section{Experimental details}

\section{Preparation of samples}

A hydrothermal synthesis method was employed for the synthesis of $\mathrm{Zn}_{1-x} \mathrm{Co}_{x} \mathrm{~S}$ NWs with nominal compositions $(x=$ $0.00,0.02,0.06,0.08,0.10)$. In a typical synthesis process, a $100 \mathrm{ml}$ Teflon-lined stainless steel autoclave was used. All of the chemicals were analytical grade and used without any further purification. The appropriate amount of zinc nitrate (10 $\mathrm{mM})$, cobalt nitrate $(10 x) \mathrm{mM}$, and thiourea $(20 \mathrm{mM})$ were dissolved in a $75 \mathrm{ml}$ deionized (DI) water and ethylenediamine ( $1: 1$ ratio) solution. Then, the mixture was stirred for $20 \mathrm{~min}$ at room temperature and these transparent solutions were transferred to an autoclave. The autoclave was sealed and kept on a hot plate at a temperature of $185^{\circ} \mathrm{C}$ for $12 \mathrm{~h}$ and then allowed to cool naturally. The obtained product was purified, washed several times by ethanol and DI water, and then filtered. The final product was dried in vacuum at $60{ }^{\circ} \mathrm{C}$ for $2 \mathrm{~h}$.

\section{Samples characterizations}

The as-synthesized samples were characterized using different techniques, such as SCXRPD. Raman spectra were obtained using a micro-Raman system from Jobin Yvon Horiba LABRAM$\mathrm{HR}$ in the visible region (400-1100 nm). Field emission scanning electron microscopy (FE-SEM) images and energydispersive spectra (EDS) were collected using the JEOL JSM6360 SEM instrument. Transmission electron microscopy (TEM) measurements were employed with an accelerating voltage of $200 \mathrm{kV}$, while selected-area electron diffraction (SAED) patterns were carried out with a Tecnai $\mathrm{G}^{2} 20$ at an accelerating voltage of $200 \mathrm{kV}$. Fourier transform infrared (FTIR) absorption spectra were obtained using the Bruker Vertex 70 spectrometer. UV-Vis absorption spectra were obtained using a Perkin-Elmer Lambda-950 UV-Vis spectrophotometer. Electron spectroscopy for chemical analysis (ESCA) was performed with X-ray photon energy of $\mathrm{Al} \mathrm{K \alpha :} 1486.61 \mathrm{eV}, 13$ $\mathrm{kV}$, and $300 \mathrm{~W}$ using an ESCA spectrometer (SPECS Surface Nano Analysis GmbH, Germany).

\section{Synchrotron radiation scattering experiments}

Synchrotron X-ray powder diffraction patterns were recorded with mode angle dispersive X-ray diffraction (ADXRD) from the beamline (BL-11) at INDUS-2, Raja Ramanna Center for Advanced Technology (RRCAT) Indore, India, at room temperature and atmospheric pressure. The SCXRPD patterns of nanowires were produced using a wavelength of $0.378377 \AA$ with energy of $\sim 33 \mathrm{keV}$. All the samples were sealed in kapton foils for the SCXRPD measurements. The 2D images were recorded using a 2D image plate (IP) camera MAR-345 having a diameter of $345 \mathrm{~mm}$. The sample was mounted orthogonally in the path of beam having a distance between the samples and detector of $211 \mathrm{~mm}$. The IP was exposed for each sample at $100 \mathrm{~s}$ and then a long $5400 \mathrm{~s}$ to obtain the total scattering data signals. These 
2D images were calibrated using a standard $\mathrm{CeO}_{2}$ sample. The obtained scattering signal of the samples was measured independently and subtracted from the background (kapton foils, air) in the data. Then, the collected 2D diffraction images were combined and subjected to geometric correction, before being integrated and converted to intensity verses $2 \theta$ plots using the FIT2D program.

\section{Results and discussion}

\section{Synchrotron X-ray powder diffraction study}

The synchrotron X-ray powder diffraction (SCXRPD) patterns of the pure and Co-doped ZnS NWs are shown in Fig. 1. The powder diffraction data were collected at a higher wave vector $Q$ $=10.2 \AA^{-1}$, which was recorded in the transmission mode using a shorter X-ray wavelength of $0.378377 \AA$ at high energy $\sim 33 \mathrm{keV}$ from beamline BL-11, $\mathrm{AD} / \mathrm{ED}-\mathrm{XRD}$ at RRCAT, Indore. The SCXRPD measurements were achieved with a highly monochromatic synchrotron beam using a Si (111) channel cut monochromator. The full scan SCXRPD is provided in the ESI Fig. S3. $\dagger$

SCXRPD revealed the high resolution diffraction for the structural refinement. The entire diffraction peaks of the sample can be readily indexed as a wurtzite structure with the JCPDS card no. 36-1450. Secondary phases and extra peaks of the alloy or sulfide phases were detected in the case of the Codoped ZnS samples due to the enhancement in solubility limit. It can be clearly observed from Fig. 2 that the peaks (200) and (210) for the CoS phases have a cubic structure and its plane values are indexed in accordance with the JCPDS card no. 830573. The intensity of the secondary phases is very low compared to the other peaks. The intensity of the secondary phase peaks for $8 \%$ Co doping is more than for $10 \%$ Co doping. When the dopant concentration reaches the maximum value of

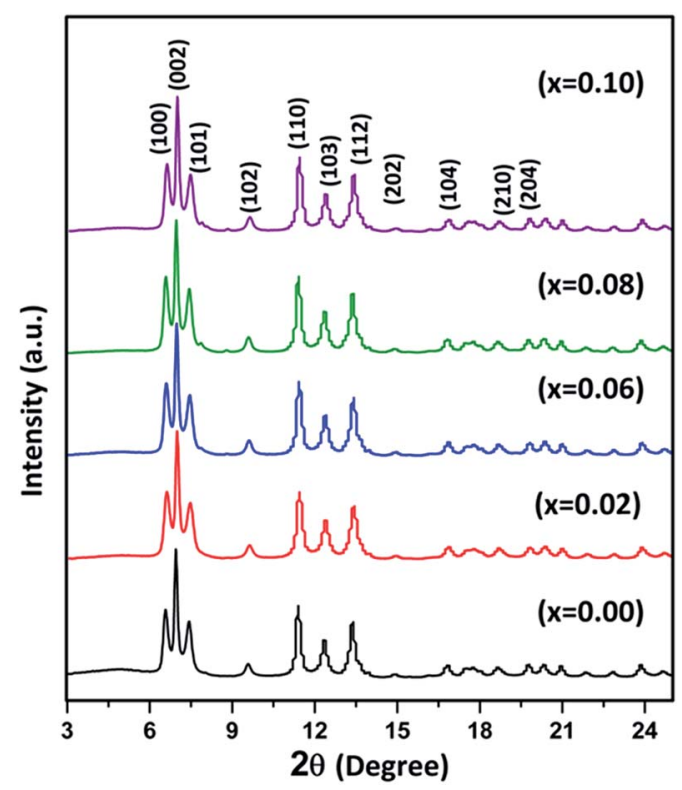

Fig. 1 SCXRPD patterns of the Co-doped ZnS NWs.

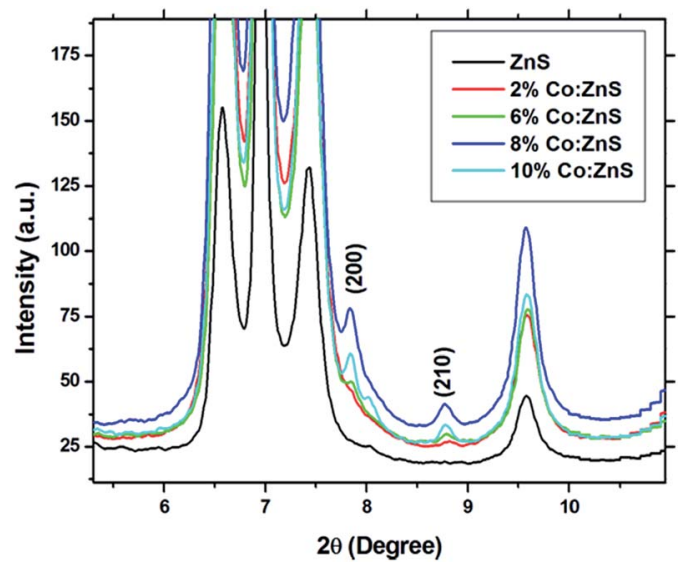

Fig. 2 Magnified view $\left(5^{\circ}-11^{\circ}\right)$ of Fig. 1 showing CoS phases with $h k l$ (200) and (210) planes in the SCXRPD patterns of the Co-doped ZnS NWs.

$10 \%$ and is reasoned as indicating an excess amount of $\mathrm{Co}^{2+}$, it readily reacts with the $\mathrm{S}^{2-}$ ions in the medium, since the solubility and ionic product constants of CoS are greater than the solubility and ionic product constants of $\mathrm{ZnS}$.

It can be noticed from Fig. 1 that the (002) peak becomes broadened in all the cases, and also this broadening can clearly be seen in Fig. 2 when this peak was magnified along with the relative intensity of peak (002), suggesting that the crystallinity of $\mathrm{ZnS}$ was degraded as a result of Co doping. It also indicates that the nanowires are grown in the (002) direction, which is further evidenced by the TEM analysis. The lattice constant, volume of unit cell, and X-ray density were calculated using the SCXRPD data and their values are plotted in Fig. 3 . The volume of the unit cell increased while the X-ray density decreased in the pure and cobalt-doped $\mathrm{ZnS}$ samples. The values of $2 \theta$ in the XRD were shifted toward a lower angle side, which reflects the lattice expansion in the crystals. The lattice expansion can also be observed when the $\mathrm{Co}^{2+}$ ions substitute for the $\mathrm{Zn}^{2+}$ sites, since the ionic radius of the $\mathrm{Co}^{2+}$ ions $(0.79 \AA)$ is larger than that of the $\mathrm{Zn}^{2+}$ ions $(0.75 \AA)$.

\section{Atomic pair distribution function analysis}

In order to obtain information on the local structure of the pure and Co-doped ZnS nanowires, SCXRPD and PDF techniques were employed in combination. The SCXRPD data were employed to obtain the PDF from the AD/ED-XRD BL-11 beamline at RRCAT, Indore. It can be seen in Fig. 4 that the as-synthesized nanowires show a series of well-defined peaks up to quite long real space distance, which means that the nanowires exhibit a medium-range periodic atomic order. ${ }^{16,30,37}$ The SCXRD patterns of the crystalline pure and Co-doped ZnS show well-defined Braggs peaks at $Q=10.2 \AA^{-1}$. For manifestation of the pair distribution functions, the obtained data were corrected and normalized using the program PDFGetX3-1.1. ${ }^{38,39}$ The manifestation of the pair distribution function $G(r)$ is the probability of finding a nearest neighbor at a certain distance ' $r$ ' between atoms. ${ }^{40,41}$ From the PDF patterns, spheralite and 

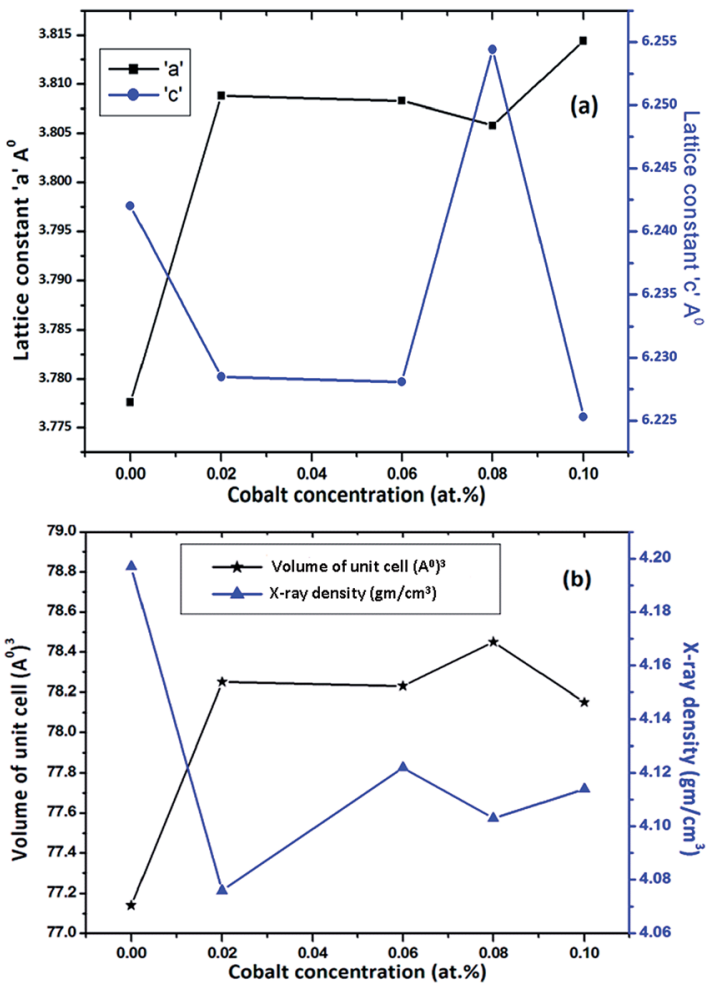

Fig. 3 (a) Lattice constant and (b) volume of unit cell and X-ray density for the pure and cobalt-doped ZnS NWs.

hexagonal structures were confirmed with the space groups $F \overline{4} 3 m$ and $P 6_{3} m c$, respectively. Sulfide and selenide semiconductor materials show two well-known structures, namely zinc blende and wurtzite structures, with identical shared tetrahedral bonding for different stacking sequences. The closepacked stacking sequence for the wurtzite structure is ABA$\mathrm{BAB} .$. along the (001) orientation whereas for the zinc blende structure the close-packed stacking sequence is ABCABC... along the (111) orientation. Yang et al. ${ }^{43}$ and Masadeh et al. ${ }^{44}$ reported that CdSe nanoparticles show a mixed state of crystalline structures between zinc blende and wurtzite structures. ${ }^{42}$

We obtained both the phases in the pure and Co-doped ZnS NWs after refinement/modeling due to inhomogeneity in the crystal structure at the nanoscale with constrain isotropic ADPs for the spheralite structure and anisotropic ADPs for the wurtzite structure. Also the results for the modeling of structures of the samples with respect to the unit cell parameter, ADPs, $z$ coordinate of $\mathrm{S}$, scale factor, resolution damping factor $\left(Q_{\mathrm{damp}}\right)$, and resolution peak broadening factor $\left(Q_{\text {broad }}\right)$ are depicted in Table 1. The sharp and well-resolved peaks in PDF suggest a high symmetry and a well-defined local structure of the NW samples. These peaks are broader, reflecting the presence of a considerable local structural disorder and strain in the assynthesized samples. The PDFs are shown in Fig. 4, which also gives the interatomic distances from the peak values. From Fig. 5, the first nearest cation-anion, second cation-cation, and third anion-anion neighboring distances of the pure and Codoped ZnS NWs are tabulated in Table 1. The cation-anion

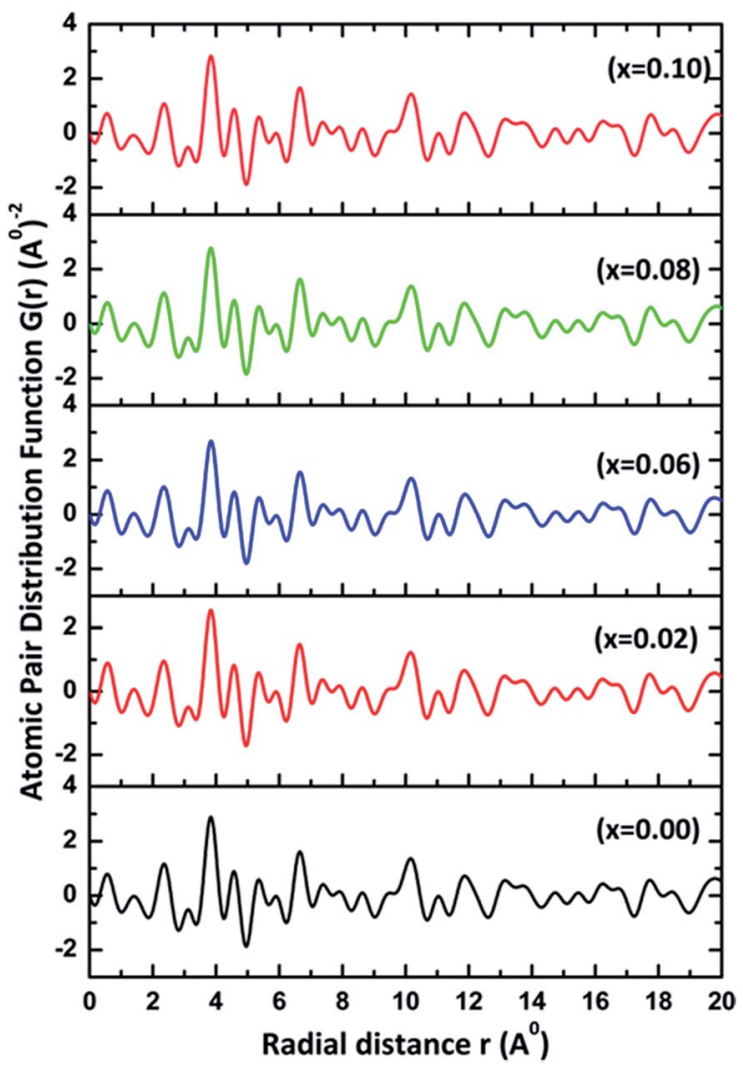

Fig. 4 The experimental pair distribution function $G(r)$ of the pure and Co-doped ZnS NWs.

distance of the $\mathrm{Zn}^{2+}-\mathrm{S}^{2-}$ bonded pair is between 2.3050 and $2.3369 \AA$ for the hexagonal and 2.3466 to $2.3511 \AA$ for the spheralite covalently bonded pair in the samples. From the first neighbor, it can be predicted that these bond length values are highly correlated. The second strong and intense peak is of the metal-metal bonding distance at 3.7733 to $3.835 \AA$, which reflects the cation-cation $\mathrm{Zn}^{2+} / \mathrm{Co}^{2+}-\mathrm{Zn}^{2+}$ neighbors of the pure and Co-doped ZnS samples. It was visualized that the finite particle size or diameter is evident in a fall-off in the intensity of the structural features with increasing the radial distance. The average diameters of the pure and Co-doped ZnS NWs were also extracted from the refined PDF and are depicted in Table 1. Quantitative structural information can be extracted from refinement of PDF by comprising data from the observed PDF and the calculated PDF from the models. The PDFgui ${ }^{53}$ computer program was used to fit and simulate the structural model to the experimental PDF, as shown in Fig. 5. Fig. 5 reflects the wurtzite structure over the wurtzite modeling using isotropic ADPs. These give a best fit for structures from the experimental and calculated PDFs from refinement, which is in agreement with the residual function. It was corroborated from Fig. 5 the good fit of the wurtzite structure model over the wurtzite structure. In Fig. 5, we observed some peaks matched to a certain extent of ' $r$ ', which means that there is possibility of multi-phases for the same structures at certain neighboring distance. The values of the refined parameters are summarized in Table 1 for their respective fit manifested in Fig. 5. The 
Table 1 The refined parameters of the pure and Co-doped ZnS NWs obtained using PDF analysis

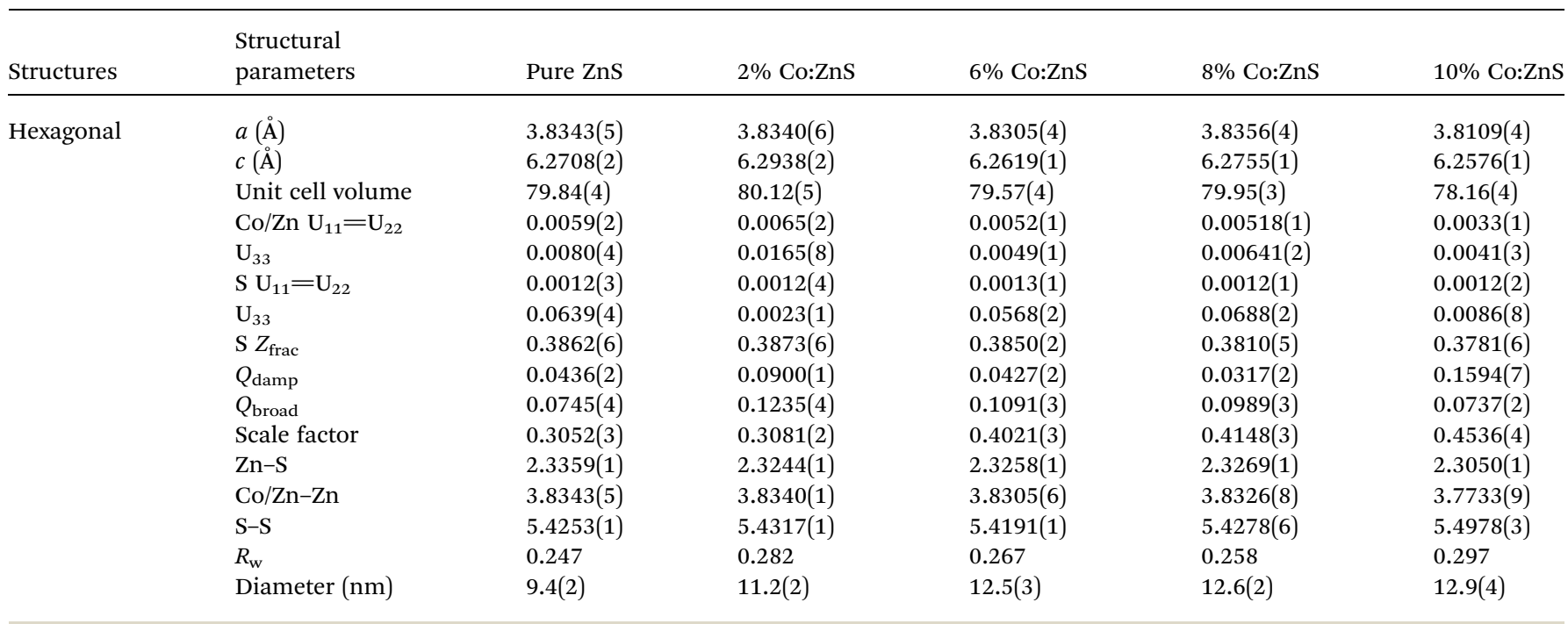

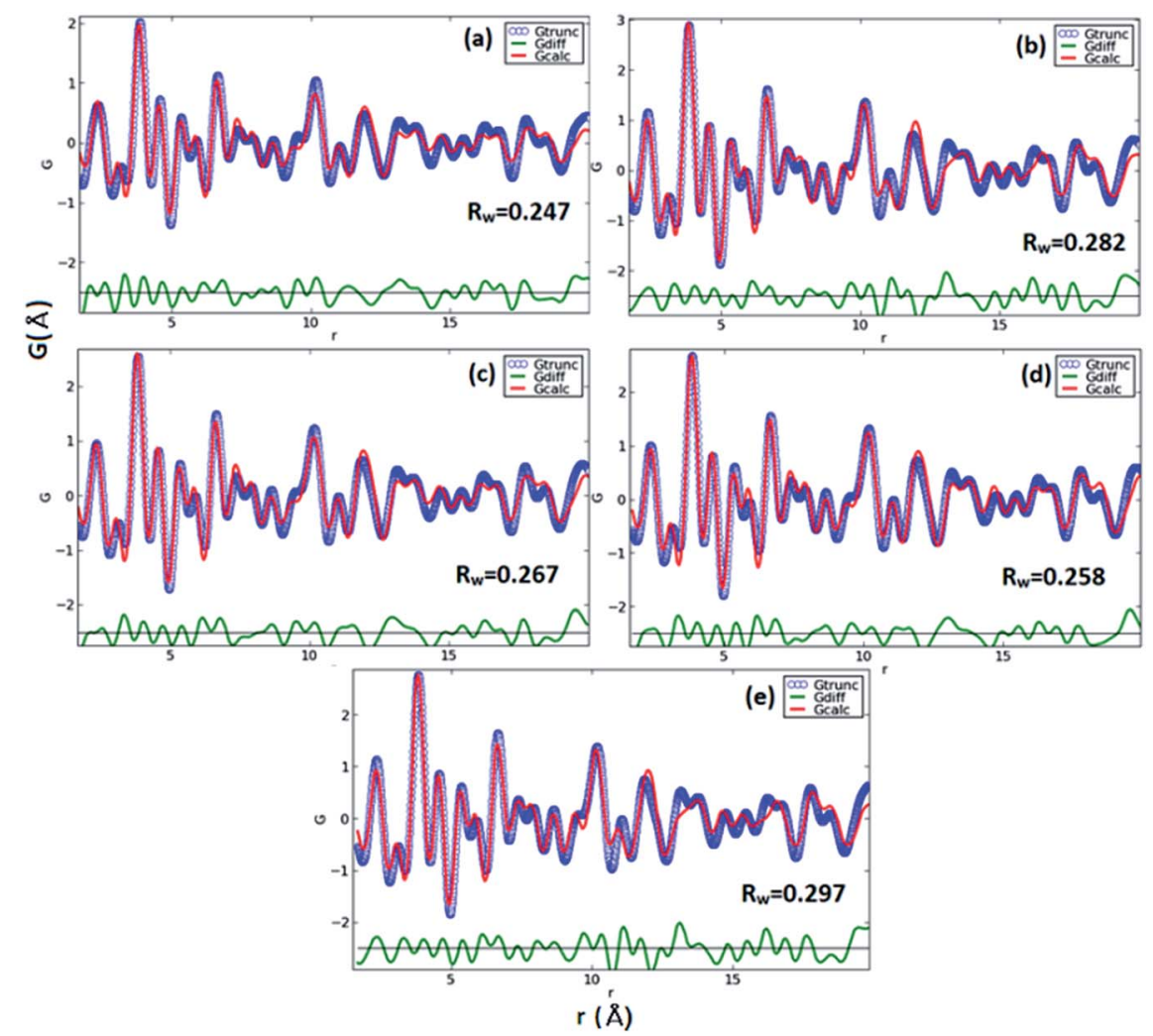

Fig. 5 The experimental PDF G(r) and calculated refined PDF with the difference curve offset given below. PDF refinement fitting using the wurtzite structure (a)-(e) model with the space group $P 6_{3} m c$ and anisotropic ADPs of the pure and Co-doped ZnS NWs, respectively.

refinement of the calculated PDF to the experimental PDF was characterized by the residual function $\left(R_{\mathrm{w}}\right)^{43}$ using eqn (1):

$$
R_{\mathrm{w}}=\sqrt{\frac{\sum_{i=1}^{N} \omega\left(r_{i}\right)\left[G_{\mathrm{obs}}\left(r_{\mathrm{i}}\right)-G_{\mathrm{calc}}\left(r_{\mathrm{i}}\right)\right]^{2}}{\sum_{i=1}^{N} \omega\left(r_{\mathrm{i}}\right) G_{\mathrm{obs}}^{2}\left(r_{\mathrm{i}}\right)}}
$$

where $G_{\text {obs }}$ is the PDF extracted from the diffraction data, $G_{\text {cal }}$ is the PDF calculated from the model, and $\omega\left(r_{\mathrm{i}}\right)$ is the weight. The structural parameters of the model were the unit cell parameter, and the anisotropic atomic displacements (ADPs) for $\mathrm{Zn}$ and S. A generated simulated PDF from calculated data which is well matched with the experimental data. The resulting very low residual signal intensity across the fitting range for pure and 
Co-doped ZnS NWs for hexagonal structure showed $R_{\mathrm{w}}=0.247$, $0.282,0.267,0.258$, and 0.297 (with the fitting range of 1.7 to 20 $\AA)$, respectively. The $R_{\mathrm{w}}$ values and their respective refined parameter results are listed in Table 1 . Fig. 5 explicitly shows the wurtzite model, which gives a better fit. The as-synthesized NWs structures do not purely belong to wurtzite or spheralite structures, but reflect intermediate structures/mixed structures.

The strain in nanostructures has been observed by different researchers, where alternative techniques were used to investigate the size-induced strain in nanostructured materials. ${ }^{43}$ The atomic structure of Co-doped ZnS NWs was subjected to different types of structural distortions relative to the corresponding pure ZnS NW materials. This approach was based on the first PDF-peak analysis, in terms of the position and width, where the local bonding of the basic building unit was investigated as a function of the nanowire diameter. The width and position could be extracted by fitting Gaussian functions, ${ }^{44}$ even without any knowledge about the adopted symmetry or atomic structure type. The first local bonding of the cation and anion of the tetrahedral $\left(\mathrm{Zn}^{2+} / \mathrm{Co}^{2+}-\mathrm{S}^{2-}\right)$ building unit was investigated $v s$. the nanoparticle diameter. The nearest first neighbor peaks at $r=2.336 \AA$ come from covalently bonded $\mathrm{Zn}^{2+}-\mathrm{S}^{2-}$ pairs. The positions and the width of these peaks were determined by fitting a Gaussian plot of ESI Fig. S4 $\dagger$ and the results are presented in Table 2 . The results outline that there is an interesting compressive strain on this nearest-neighbor bond length, which is possible to measure with the PDF in a high accuracy. It was observed that internal strain deceases rapidly with an enhancing NW diameter. The bond length of $\mathrm{Zn}-\mathrm{S}$ pairs shortens as the NWs diameters enhance, which suggest the presence of an internal stress in the NWs. The $\mathrm{Zn}-\mathrm{S}$ bond lengths extracted from the PDF structural refinement were also in good agreement with those obtained from the first-peak Gaussian fit, as shown in the ESI Fig. S4. $\dagger$ Apparently, there was no size-dependent homogeneous strain measurable on the first peak.

\section{Micro-Raman spectroscopic study}

Micro-Raman measurements of the as-synthesized samples were carried out using an excitation laser wavelength of argon $488 \mathrm{~nm}$ to study the effects of the cobalt-substituted ZnS NWs. The background subtracted/corrections Raman spectra data for the as-synthesized NWs are shown in Fig. 6. It is known that the wurtzite $\mathrm{ZnS}$ belongs to the symmetric space group $C_{6 v}^{4}\left(P 6_{3} m c\right)$ in the primitive cell. The zone-center optical phonon can be classified as the following irreducible representation:

$$
\Gamma_{\mathrm{opt}}=\mathrm{A}_{1}+\mathrm{E}_{1}+2 \mathrm{E}_{2}+2 \mathrm{~B}_{1}
$$
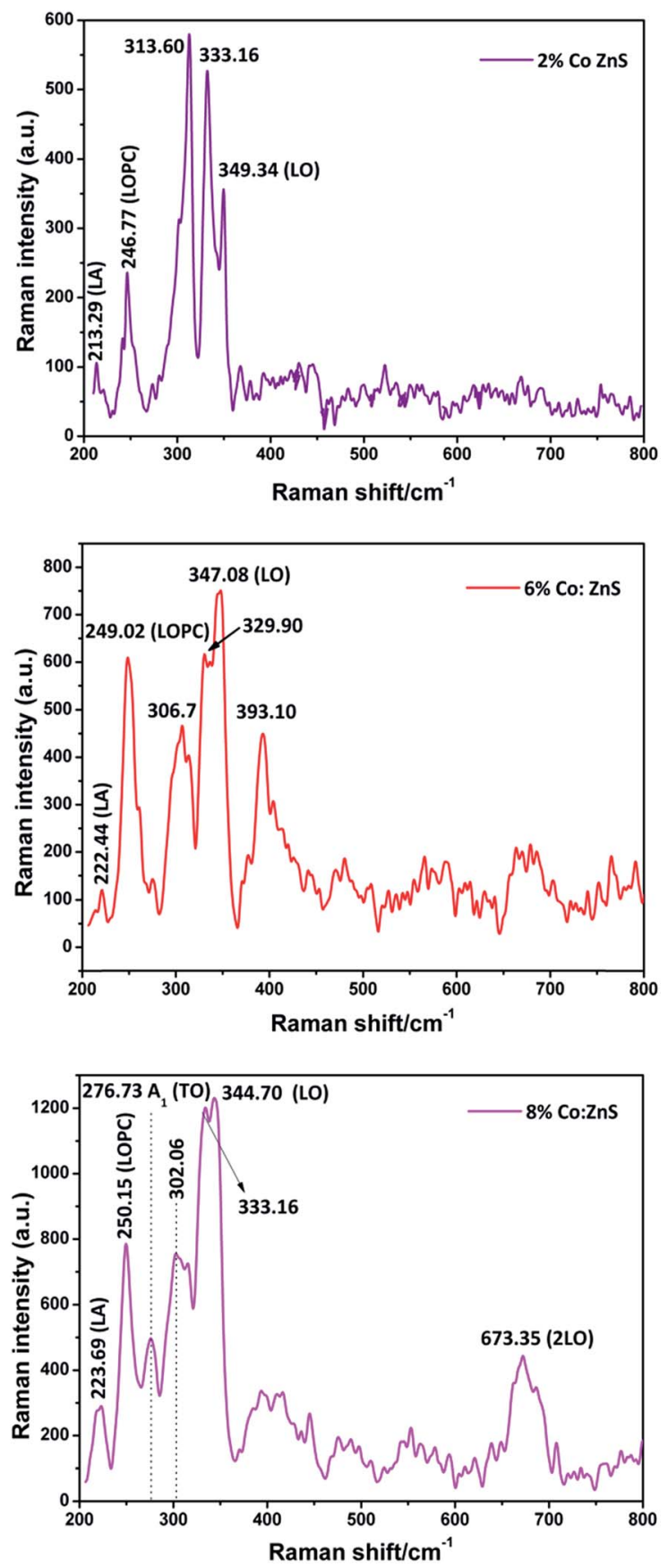

Fig. 6 Room temperature micro-Raman spectra of first-order TO and LO phonons modes for $2 \%, 6 \%, 8 \% \mathrm{Co}: \mathrm{ZnS} N W \mathrm{~s}$. The genuine feature of a spectral line observed at $\sim 250 \mathrm{~cm}^{-1}$ for the LOPC mode.

where, $\mathrm{A}_{1}$ and $\mathrm{E}_{1}$ are polar modes and both are Raman and infrared active, while the $\mathrm{E}_{2}$ modes are nonpolar and Raman active only and the $\mathrm{B}_{1}$ modes are silent modes. The longitudinal

Table 2 The first PDF-peak position (FPP) and width (FPW) for the pure and Co-doped ZnS nanowires

\begin{tabular}{|c|c|c|c|c|c|}
\hline & Pure ZnS & $2 \%$ Co: $Z n S$ & $6 \%$ Co: $Z n S$ & $8 \%$ Co: $\mathrm{ZnS}$ & 10\% Co:ZnS \\
\hline PDF FPP & 2.3375 & 2.3320 & 2.3286 & 2.3232 & 2.3173 \\
\hline PDF FPW & 0.4451 & 0.4359 & 0.4449 & 0.4515 & 0.4575 \\
\hline
\end{tabular}


optic (LO) peaks have been reported to center at around 350 $\mathrm{cm}^{-1}$ for both hexagonal and cubic structures of $\mathrm{ZnS} .^{45}$ For the samples of $\mathrm{Zn}_{1-x} \mathrm{Co}_{x} \mathrm{~S}$ of NWs with Co concentrations at $x=$ $0.02,0.06$, and 0.08 , sharp peaks could be clearly observed at 246.77, 249.02, and 250.15 respectively. Jimenez-Sandoval et al. $^{46}$ reported 3 peaks in the range of spectral region, i.e., transverse optic (TO) (276) to LO $\left(350 \mathrm{~cm}^{-1}\right)$ for the TM $\left(\mathrm{Co}^{2+}\right.$, $\mathrm{Fe}^{2+}, \mathrm{Mn}^{2+}$, etc.)-substituted $\mathrm{ZnS}$ nanomaterials. In this article, only two peaks appeared in that region at $313.60,333.16 \mathrm{~cm}^{-1}$, and $306.7,329.90 \mathrm{~cm}^{-1}$, while the peak at $300 \mathrm{~cm}^{-1}$ was not observed for 0.02 and 0.06 respectively. In the case of $8 \% \mathrm{Co}-$ doped $\mathrm{ZnS}$, peaks at 333.16 and $302.06 \mathrm{~cm}^{-1}$ were observed. Furthermore, for all the samples, the peaks at 349.34, 347.08, and $344.70 \mathrm{~cm}^{-1}$ were assigned to strong first-order scattering from an unresolved doublet, which identifies the $A_{1}$ and $E_{1}$ symmetry LO phonon vibration modes. It was confirmed the $\mathrm{E}_{1}$ (LO) mode results from the lateral morphology of the Co-doped $\mathrm{ZnS}$ nanowires parallel to the $c$-axis, ${ }^{47}$ and in addition, strong surface optical (SO) phonon modes were observed at 330 and $333 \mathrm{~cm}^{-1} \cdot{ }^{45-47}$ As compared to the Raman spectrum of bulk hexagonal $\mathrm{ZnS}$ (LO: $352 \mathrm{~cm}^{-1}$ ) (Brafman et $a l^{48}$ and Nilsen et $a l .{ }^{49}$ ), the peaks of the first-order LO phonons from these $\mathrm{ZnS}$ nanowires exhibit a shift toward lower energy. The red-shift of the LO mode in the samples indicates that a tensile strain existed in the lattice. Due to the limited solubility of Co ions in $\mathrm{ZnS}$ (2\%), it was expected the Co ions would be located interstitially in the lattice instead of being substituted for $\mathrm{Zn}^{2+}$ as the Co concentration increased. Therefore, the gradual shift of the LO mode as well as the tensile strain with increasing Co content are believed to result from the excess incorporation of Co interstitially in the ZnS lattice. This strain effect could also be validated by observation of the broadening LO peak value with the increasing Co content, as shown in Fig. 6. This also confirmed the strain of the samples using the PDF analysis given in the ESI Fig. S4. $\uparrow$ The minor peaks were clearly identified for the mode at $\sim 213.29,222.44$ and $223.69 \mathrm{~cm}^{-1}$ corresponding to the second-order longitudinal acoustic (2LA) phonon in Co:ZnS NWs ${ }^{45}$ together with a sharp peak at $276.73 \mathrm{~cm}^{-1}$, which consists of the assigned modes of $\mathrm{A}_{1}$ (TO). It is also observed that the broad peak at 673.35 in $8 \%$ Co:ZnS NWs could be assigned to second-order LO. Kumar et $a l^{50}$ reported that the coupling between longitudinal phonons and charge density modes, namely $\mathrm{L}^{+}$and $\mathrm{L}^{-}$modes, were detected. As the charge carrier concentration in the semiconductor increases, the frequency of the $\mathrm{L}^{+}$mode increases from bulk LO mode frequency, while the frequency of the $\mathrm{L}^{-}$mode increases from zero to the TO mode frequency. ${ }^{50}$ Hence the peaks at $246.8,249$, and $250.15 \mathrm{~cm}^{-1}$ observed in the present samples can be tentatively attributed to the LOPC $\left(\mathrm{L}^{-}\right)$mode, ${ }^{57}$ which may be due to the formation of a small portion of the intermediate state due to the uncompleted conversion during the synthesis process. It can be observed from Fig. 6 that the LO mode is more altered while the peak position of the LOPC mode has shifted to higher frequencies with the increase in cobalt concentrations, which is attributed to the decrease in carrier concentration, which reveals the existence of free electrons by $\mathrm{Co}^{+}$ions. In addition, second-order Raman scattering is seen in the range of $350-450 \mathrm{~cm}^{-1}$. This second- order scattering consist of a TO + transverse acoustic (TA) phonon mode at $\sim 393 \mathrm{~cm}^{-1}$ for $6 \%$ Co:ZnS NWs. It is similar to the 2LA phonon response and multiphonon response in the range of $350-450 \mathrm{~cm}^{-1}$ which is observed because the strong plasmons background fluctuations in polar semiconducting systems result in two different LOPC modes.

\section{Morphological study}

The nanowires assembled into micrometer-diameter-sized spheres or flowers of the pure and Co-doped ZnS samples are shown in Fig. 7. Fig. 8 shows the micrographs of the assynthesized samples, where it is quite clear that the NWs structure is assembled into micro-flowers with a high aspect ratio. TEM images of the as-synthesized samples are shown in Fig. 8. The TEM micrographs show the extremely uniform diameter of the nanowire micro-flowers. The average diameters of the pure and Co-doped ZnS NWs were found to be 8.18, 12.31, 12.71 , and $14.44 \mathrm{~nm}$ with lengths of tens of micrometers respectively. The average diameter of the undoped and Codoped ZnS micro-flowers was evaluated to be $1200 \mathrm{~nm}$ using FE-SEM and TEM micrographs. Selected-area electron diffraction (SAED) pattern were taken from the nanowires of the microflowers of $6 \%$ Co:ZnS, which suggest it has a single crystal nature. The spots on the SAED patterns can be easily attributed to the $[110]$ zone axis of the nanowires. The compositional

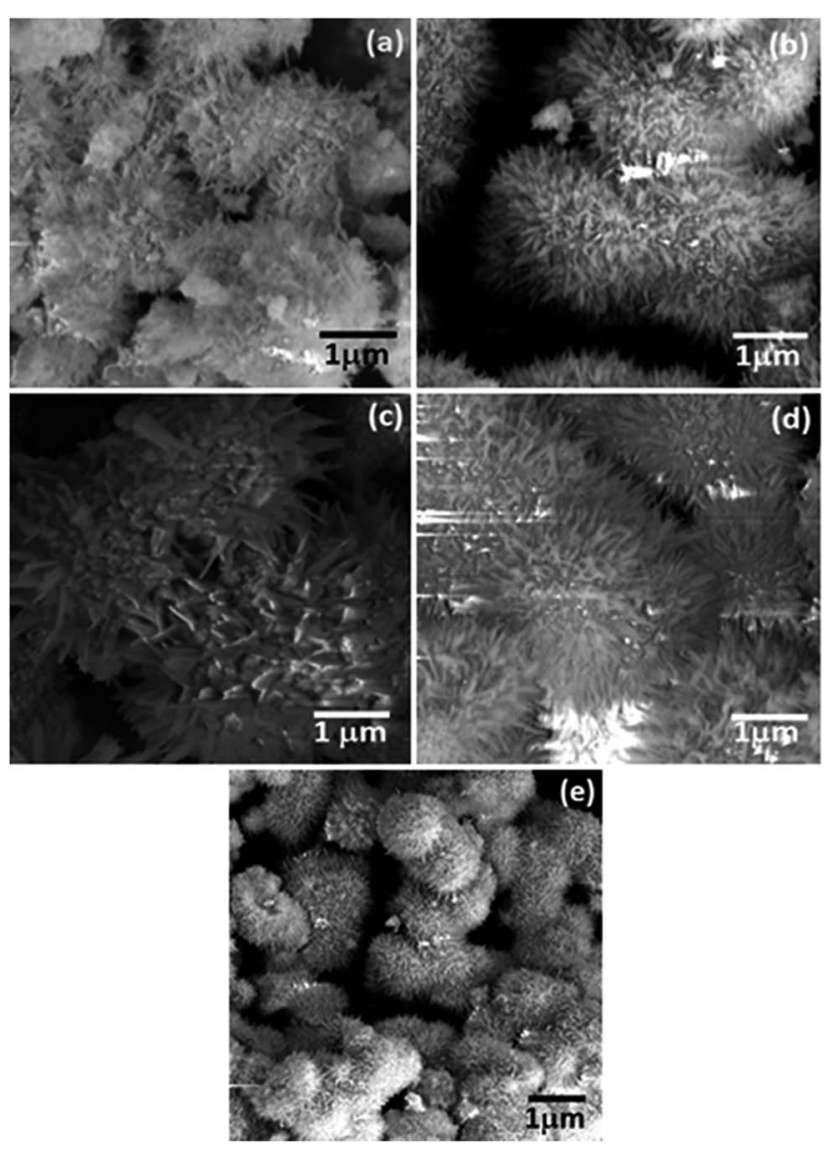

Fig. 7 (a-e) FE-SEM images of the pure and Co-doped ZnS NWs. 

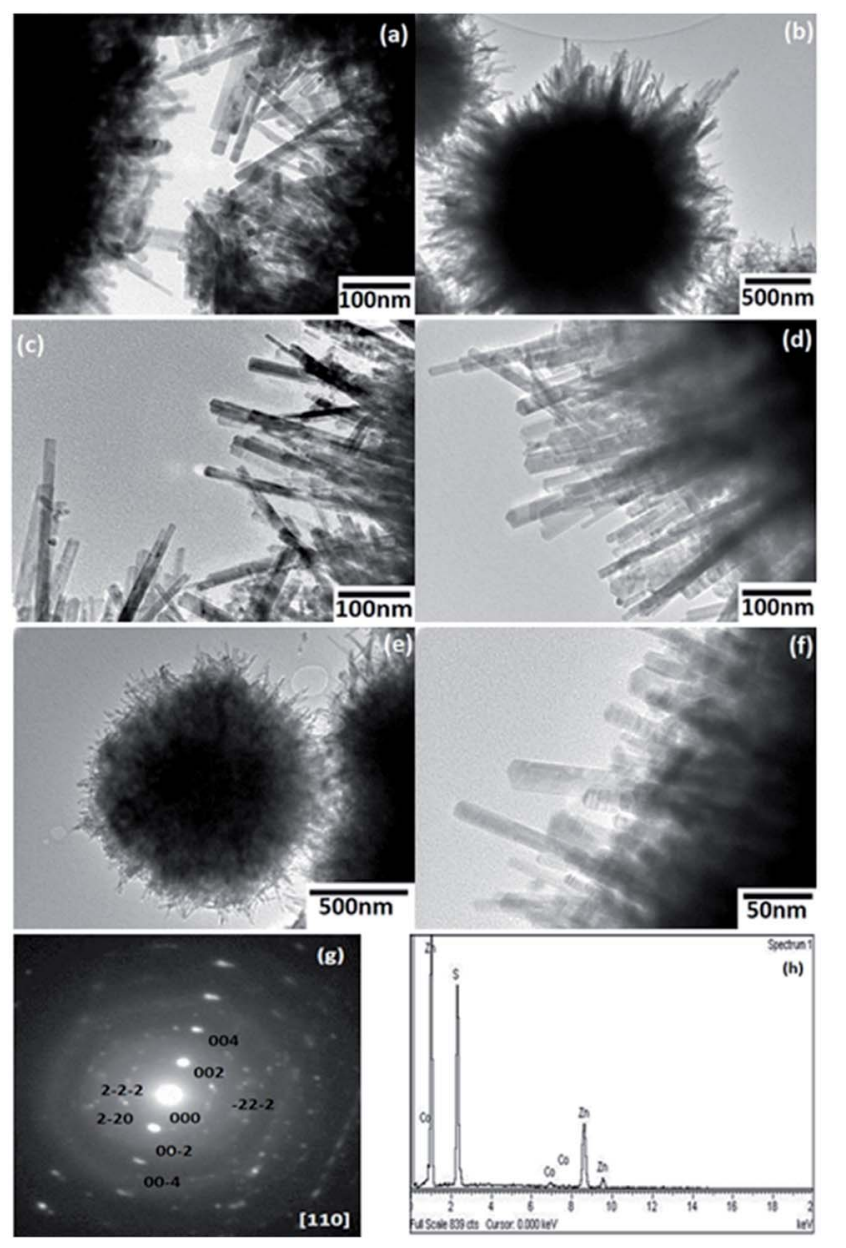

Fig. 8 TEM images of the Co-doped ZnS NWs as (a) and (b) images for pure ZnS (c) for $0.06 \mathrm{Co:ZnS,} \mathrm{(d),} \mathrm{(e)} \mathrm{for} 0.08 \mathrm{Co:ZnS,} \mathrm{(f)} \mathrm{for} 0.10$, (g) and (h) SAED pattern and EDAX spectra corresponding to $0.06 \mathrm{Co}: \mathrm{ZnS}$ micro-flower nanowires.

analysis of the pure and Co-doped ZnS NWs was obtained from the energy-dispersive absorption X-ray (EDAX) and XPS spectra of the samples, as shown in the ESI Fig. S1 and S2, $\uparrow$ respectively, and the FTIR spectra are also given in the ESI Fig. $55 ; \dagger$ it can be noticed that the peaks of $\mathrm{Zn}, \mathrm{S}$, and Co are present. For the pure $\mathrm{ZnS}$ structure, qualitative analysis reveals that the atomic ratio of $\mathrm{Zn}$ to $\mathrm{S}$ is very close to $1: 1$ stoichiometrically. For $2 \%$ Codoped ZnS NWs, the atomic\% of $\mathrm{Zn}$, Co, and $\mathrm{S}$ was found to be $50.55,0.63$, and 48.82 , respectively, whereas for $6 \%$ and $10 \%$ Co:ZnS NWs the atomic\% of the elements of $\mathrm{Zn}, \mathrm{Co}$, and $\mathrm{S}$ was found to be $50.88,2.02,46.50$ and $49.75,3.59,46.66$, respectively. The amount of Co element detected was less than that actually added during synthesis.

\section{Optical study}

The UV-visible absorption spectra of the pure and Co-doped ZnS NWs are shown in Fig. 9. The absorption peaks were observed at $385.95 \mathrm{~nm}, 416.05 \mathrm{~nm}, 420.06 \mathrm{~nm}, 438 \mathrm{~nm}$, and $448 \mathrm{~nm}$ for the pure and Co-doped ZnS NWs, respectively. A red-shift was observed at the absorption edge to the smaller wavelength side, which was due to exchange interactions between the d-electrons (a)

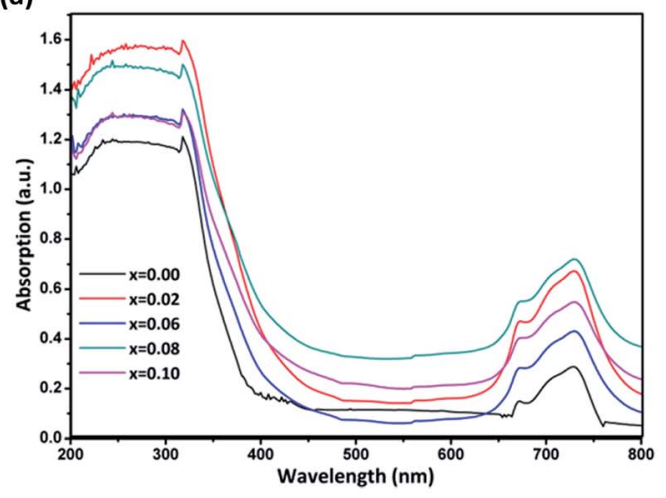

(b)

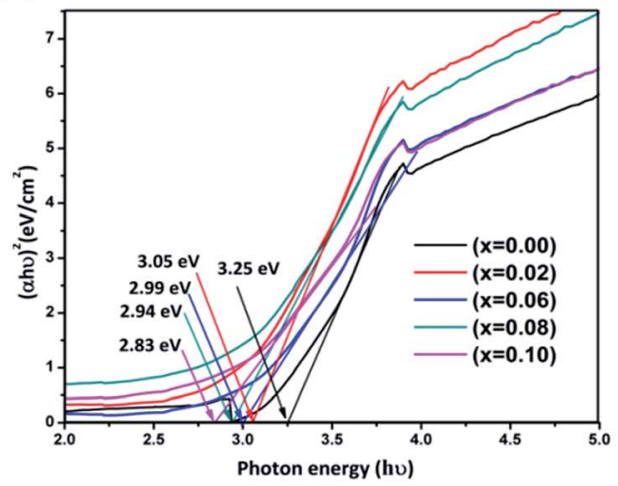

Fig. 9 (a) Absorption spectra of the pure and cobalt-doped ZnS NWs. (b) Tauc plots of the pure and cobalt-doped ZnS NWs.

of cobalt ions and the $\mathrm{s}$ and $\mathrm{p}$ electrons of the host electron band. The pure and Co-doped ZnS samples show a red-shift comparable to bulk $\mathrm{ZnS}$ (345 $\mathrm{nm}$ ). This red-shift can be attributed to the quantum confinement effect in the NWs. The energy band gap was calculated from the Tauc eqn (3): ${ }^{11}$

$$
\alpha h \nu=A\left(h \nu-E_{\mathrm{g}}\right)^{\frac{1}{2}}
$$

where $\alpha$ is the absorption coefficient, $h \nu$ is the photon energy, $A$ is the constant, and $E_{\mathrm{g}}$ is the energy band gap of the sample. $\mathrm{ZnS}$ has a direct band gap, hence the value of $n$ is $\frac{1}{2}$, because transition from the valence band to conduction band is direct. The energy band gaps of the pure and Co-doped ZnS samples were found to be $3.25,3.05,2.99,2.94$, and $2.83 \mathrm{eV}$, respectively. The $\mathrm{Co}^{2+}$ ions substitution in the $\mathrm{ZnS}$ lattice effects the sp$\mathrm{d}$ exchange interaction between the band electrons and the localized d-electron of the $\mathrm{Co}^{2+}$ ions. The calculated energy bandgap of the samples was found to be decrease with the increase in $\mathrm{Co}^{2+}$ content; this was attributed to the negative and positive correction to the conduction and valence band edges, which arise from the $\mathrm{s}-\mathrm{d}$ and $\mathrm{p}-\mathrm{d}$ interactions, respectively, resulting in shrinkage of the bandgap. ${ }^{52}$

\section{FTIR study}

FTIR transmittance spectra for the pure and Co-doped ZnS samples are shown in Fig. 10. The sharp and strong peaks also 


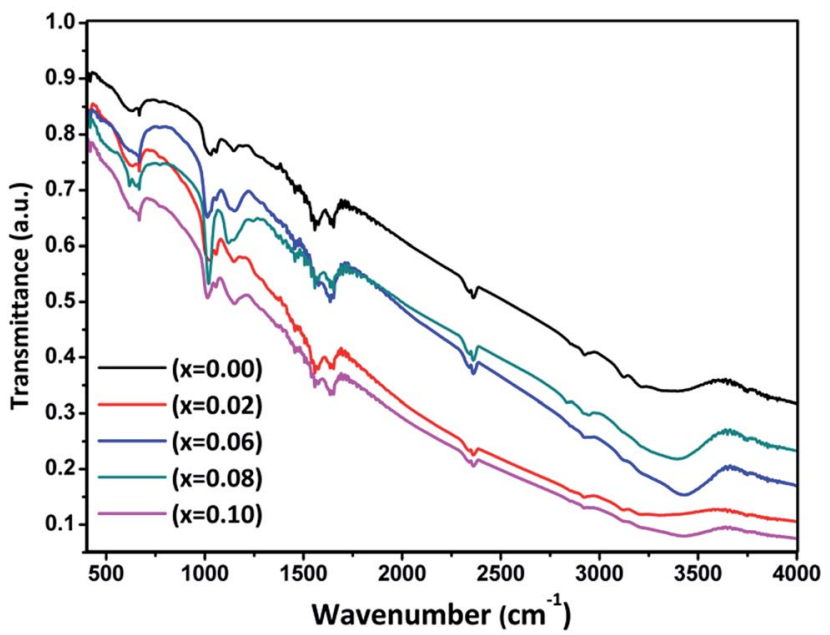

Fig. 10 FTIR spectra of the pure and Co-doped ZnS NWs.

show the samples were highly crystalline in nature. There were only a few peaks observed at $400-4000 \mathrm{~cm}^{-1}$. It is well known that an absorption peak at $400-650 \mathrm{~cm}^{-1}$ indicates $\mathrm{Zn}$ and $\mathrm{S}$ stretching; ${ }^{54}$ here, we also observed peaks for pure and Codoped ZnS NWs at 416.58, $666.30 \mathrm{~cm}^{-1} ; 419.43,670.53 \mathrm{~cm}^{-1}$; 419.33, 662; 419.43, $626.33 \mathrm{~cm}^{-1}$; and 410.97, $652.66 \mathrm{~cm}^{-1}$; respectively. This indicates the formation of pure and cobaltdoped ZnS NWs. The absorption bands at 2359 to $2923 \mathrm{~cm}^{-1}$ were attributed to $-\mathrm{C}-\mathrm{H}$ vibration, ${ }^{55}$ which were observed due to the doping of cobalt. The observed values at 3215 to $3321 \mathrm{~cm}^{-1}$ indicate $\mathrm{OH}$ stretching. The peaks at 1038.5,1163; 1027, 1150; 1011, 1150; 1019.4, $1133 \mathrm{~cm}^{-1}$; and 1011, $1158 \mathrm{~cm}^{-1}$ were observed due to the formation of micro-structures for the pure and Co-doped ZnS samples, respectively; ${ }^{55,56}$ whereas, the $-\mathrm{NH}_{2}$ stretching, scissor vibrations, and $\mathrm{C}-\mathrm{N}$ stretching vibration at 1000 to $1160 \mathrm{~cm}^{-1}$ appeared in the FTIR spectra. ${ }^{56}$ The bands at 1550 to $1658 \mathrm{~cm}^{-1}$ are due to the $-\mathrm{C}=\mathrm{O}$ stretching mode, arising from the absorption of atmospheric $\mathrm{CO}_{2}$ on the surface of the samples (Qadri et al.). ${ }^{55}$ The band values are in good agreement with those reported in the literature. ${ }^{55-58}$ The close similarity in the FTIR spectra of pure and Co-doped ZnS NWs outlines that $\mathrm{Co}$ is substituted into the $\mathrm{ZnS}$ lattice without altering the crystal structure.

\section{Conclusions}

In conclusion, the samples of pure and cobalt-doped ZnS microflower nanowires were successfully synthesized by a hydrothermal method. Synchrotron X-ray powder diffraction data were employed for analysis of the pair distribution function (PDF). The PDF was utilized to address the atomic-scale structure of the as-synthesized NWs. The interatomic distance was calculated considering the PDF analysis. Total X-ray diffraction coupled to PDF data analysis allows the determination of the presence of nanoscale inhomogeneity in the structure. The internal strain was extracted from PDF as a function diameter of the nanowires and showed that the internal strain increased with Co doping due to the enhancement in the diameter of the nanowires. It was observed from the FE-SEM and TEM image analyses that the morphology of the as-synthesized samples showed the NWs structure was assembled into micro-size flower (clusters of nanowires)-like shapes. The Raman spectra were obtained for the pure and Co-doped ZnS NWs, which exhibited second-order longitudinal acoustic (2LA) phonon modes at $\sim 213.29,222.44$, and $223.69 \mathrm{~cm}^{-1}$, first-order phonon modes at $349.34,347.08$, and $344.70 \mathrm{~cm}^{-1}$ corresponding to $\mathrm{A}_{1} / \mathrm{E}_{1}$ longitudinal-optical-phonon vibration modes, and a strong surface optical (SO) phonon mode observed at 330 to $333 \mathrm{~cm}^{-1}$. The LOPC $\left(\mathrm{L}^{-}\right)$mode was observed due to free electrons from the $\mathrm{Co}^{+}$ions. EDAX and XPS studies showed the successful doping of cobalt. The energy band gap of cobalt-doped ZnS samples showed a red-shift as compared to the bulk ZnS band gap. Also the size of nanowires increase with the increasing cobalt content, which reduced the band gap. It was confirmed from the FTIR study that cobalt substitutes to the ZnS site. The combination of synchrotron X-ray diffraction and atomic PDF shows potential as a tool for the standardization of the atomicscale structure of nanomaterials.

\section{Acknowledgements}

The authors are grateful to Prof. S. S. Shah for his encouragement. Also the authors are thankful to Dr K. K. Pandey High Pressure \&Synchrotron Radiation Physics Division, Bhabha Atomic Research Centre, Mumbai (India) for availing beamline BL-11 for SCXRPD facility, Dr N. P. Lalla and Dr U. P. Deshpande UGC-DAE CSR, University Campus, Khandwa Road, Indore (India) for providing TEM and XPS, UV-Vis, FTIR facilities.

\section{References}

1 P. Jiang, J. Jie, Y. Yu, Z. Wang, C. Xie, X. Zhang, C. Wu, L. Wang, Z. Zhu and L. Luo, J. Mater. Chem., 2012, 22, 6856.

2 C. Wu, J. Jie, L. Wang, Y. Yu, Q. Peng, X. Zhang, J. Cai, H. Guo, D. Wu and Y. Jiang, Nanotechnology, 2011, 22, 069801.

3 H. Ong and R. Chang, Appl. Phys. Lett., 2001, 79, 3612-3614. 4 T. Tran, W. Park, W. Tong, M. Kyi, B. Wagner and C. Summers, J. Appl. Phys., 1997, 81, 2803-2809.

5 Z. Zhong, Science, 2003, 302, 1377-1379.

6 Y. Cui, Z. Zhong, D. Wang, W. Wang and C. Lieber, Nano Lett., 2003, 3, 149-152.

7 O. Shirak, O. Shtempluck, V. Kotchtakov, G. Bahir and Y. Yaish, Nanotechnology, 2012, 23, 395202.

8 P. Calandra, M. Goffredi and V. Liveri, Colloids Surf., A, 1999, 160, 9-13.

9 Y. Cui, Science, 2001, 293, 1289-1292.

10 Z. Chen, L. Cheng, H. Xu, J. Liu, J. Zou, T. Sekiguchi, G. Lu and H. Cheng, Adv. Mater., 2010, 22, 2376-2380.

11 Q. Wang, B. Liu, X. Wang, S. Ran, L. Wang, D. Chen and G. Shen, J. Mater. Chem., 2012, 22, 21647.

12 M. Ruedas-Rama, A. Orte, E. Hall, J. Alvarez-Pez and E. Talavera-Rodriguez, Biophys. J., 2011, 100, 468a-469a.

13 S. Acharya, N. Maheshwari, L. Tatikondewar, A. Kshirsagar and S. Kulkarni, Cryst. Growth Des., 2013, 13, 1369-1376. 
14 M. Limaye, S. Gokhale, S. Acharya and S. Kulkarni, Nanotechnology, 2008, 19, 415602.

15 D. Wu, Y. Jiang, Y. Zhang, Y. Yu, Z. Zhu, X. Lan, F. Li, C. Wu, L. Wang and L. Luo, J. Mater. Chem., 2012, 22, 23272.

16 J. Kim, S. Kim, D. Kim, K. Oh, W. Hong, T. Bae and H. Chung, J. Nanomater., 2016, 2016, 1-6.

17 U. Gawai, H. Khawal, T. Shripathi and B. Dole, CrystEngComm, 2016, 18, 1439-1445.

18 S. Kar and S. Chaudhuri, J. Phys. Chem. B, 2005, 109, 32983302.

19 S. Acharya, S. Bhoga and K. Singh, Integr. Ferroelectr., 2010, 116, 16-22.

20 S. Pahari, A. Sinhamahapatra, N. Sutradhar, H. Bajaj and A. Panda, Chem. Commun., 2012, 48, 850-852.

21 W. Xuan-Rong, Y. Qiao-Zhen, Z. Yong-Xiang and L. Yan-Luo, J. Inorg. Mater., 2016, 31, 473.

22 M. Wei, J. Yang, Y. Yan, J. Cao, Q. Zuo, H. Fu, B. Wang and L. Fan, Superlattices Microstruct., 2013, 54, 181-187.

23 J. Cao, L. Fan, J. Yang, Y. Yan, M. Wei, L. Yang, B. Feng, D. Han, B. Wang and H. Fu, Superlattices Microstruct., 2013, 57, 58-65.

24 M. Lu, L. Chen, W. Mai and Z. Wang, Appl. Phys. Lett., 2008, 93, 242503.

$25 \mathrm{X} . \mathrm{Xu}, \mathrm{G} . \mathrm{Fei}, \mathrm{W} . \mathrm{Yu}, \mathrm{X}$. Wang, L. Chen and L. Zhang, Nanotechnology, 2005, 17, 426-429.

26 R. Chen, D. Li, B. Liu, Z. Peng, G. Gurzadyan, Q. Xiong and H. Sun, Nano Lett., 2010, 10, 4956-4961.

27 C. Bradford, C. O'Donnell, B. Urbaszek, A. Balocchi, C. Morhain, K. Prior and B. Cavenett, J. Cryst. Growth, 2001, 228, 634-638.

28 M. Navaneethan, J. Archana, K. Nisha, Y. Hayakawa, S. Ponnusamy and C. Muthamizhchelvan, Mater. Lett., 2012, 68, 78-81.

29 M. Huang, Y. Cheng, K. Pan, C. Chang, F. Shieu and H. Shih, Appl. Surf. Sci., 2012, 261, 665-670.

30 U. Gawai, H. Khawal, M. Bodke, K. Pandey, U. Deshpande, N. Lalla and B. Dole, RSC Adv., 2016, 6, 50479-50486.

31 V. Petkov, Mater. Today, 2008, 11, 28-38.

32 V. Petkov, K. Rangan, M. Kanatzidis and S. Billinge, Mater. Res. Soc. Symp. Proc., 2001, 678.

33 C. Ho, P. Varadhan, H. Wang, C. Chen, X. Fang and J. He, Nanoscale, 2016, 8, 5954-5958.

34 M. Seong, S. Chun, H. Cheong, N. Samarth and A. Mascarenhas, Phys. Rev. B: Condens. Matter Mater. Phys., 2002, 66, 033104.

35 Y. Lin, C. Chiu, W. Fan, C. Chia, S. Yang, D. Chuu, M. Lee, W. Chen, W. Chang and W. Chou, J. Appl. Phys., 2007, 102, 123510 .
36 H. Harima, S. Nakashima and T. Uemura, J. Appl. Phys., 1995, 78, 1996-2005.

37 M. Feygenson, J. Neuefeind, T. Tyson, N. Schieber and W. Han, Inorg. Chem., 2015, 54, 11226-11235.

38 V. Petkov, T. Ohta, Y. Hou and Y. Ren, J. Phys. Chem. C, 2007, 111, 714-720.

39 P. Juhás, T. Davis, C. Farrow and S. Billinge, J. Appl. Crystallogr., 2013, 46, 560-566.

40 K. Page, T. Hood, T. Proffen and R. Neder, J. Appl. Crystallogr., 2011, 44, 327-336.

41 S. Billinge and M. Kanatzidis, ChemInform, 2004, 35, 345360.

42 T. Egami and S. Billinge, Mater. Today, 2003, 6, 57.

43 X. Yang, A. Masadeh, J. McBride, E. Božin, S. Rosenthal and S. Billinge, Phys. Chem. Chem. Phys., 2013, 15, 8480.

44 A. Masadeh, E. Božin, C. Farrow, G. Paglia, P. Juhas, S. Billinge, A. Karkamkar and M. Kanatzidis, Phys. Rev. B: Condens. Matter Mater. Phys., 2007, 76, 113101.

45 J. Kim, H. Rho, J. Kim, Y. Choi and J. Park, J. Raman Spectrosc., 2012, 43, 906-910.

46 S. Jiménez-Sandoval, A. López-Rivera and J. Irwin, Phys. Rev. B: Condens. Matter Mater. Phys., 2003, 68, 052101.

47 H. Lu, S. Chu and C. Chang, J. Cryst. Growth, 2005, 280, 173178.

48 O. Brafman and S. Mitra, Phys. Rev., 1968, 171, 931-934.

49 W. Nilsen, Phys. Rev., 1969, 182, 838-850.

50 S. Kumar, M. Khadar, S. Dhara, T. Ravindran and K. Nair, Nucl. Instrum. Methods Phys. Res., Sect. B, 2006, 251, 435-440.

51 J. Tauc, R. Grigorovici and A. Vancu, Phys. Status Solidi B, 1966, 15, 627-637.

52 L. Li, H. Liu, X. Luo, X. Zhang, W. Wang, Y. Cheng and Q. Song, Solid State Commun., 2008, 146, 420-424.

53 C. Farrow, P. Juhas, J. Liu, D. Bryndin, E. Božin, J. Bloch, T. Proffen and S. Billinge, J. Phys.: Condens. Matter, 2007, 19, 335219.

54 S. Ummartyotin, N. Bunnak, J. Juntaro, M. Sain and H. Manuspiya, Solid State Sci., 2012, 14, 299-304.

55 A. Mandal, A. Dandapat and G. De, Analyst, 2012, 137, 765772.

56 S. Qadri, E. Skelton, D. Hsu, A. Dinsmore, J. Yang, H. Gray and B. Ratna, Phys. Rev. B: Condens. Matter Mater. Phys., 1999, 60, 9191-9193.

57 J. Wang, T. You, H. Feng, K. Chen and B. Xu, J. Cryst. Growth, 2013, 374, 60-64.

58 U. Gawai, U. Deshpande and B. Dole, $R S C A d v$., 2017, 7, 12382-12390. 